\title{
Extended studies on fretting wear criterion for coated systems with complete contact based on accumulated specific friction energy
}

\author{
E. Leidich, A. Maiwald \& J. Vidner \\ Chemnitz University of Technology, Germany
}

\begin{abstract}
This paper presents the results of basic studies investigating fretting behaviour in different coated steel-pairings. Performed on a test bench for the determination of friction coefficients using a standardized torsion test method, the completed experiments enabled the identification of variations in the temporal and tribological behaviour of each pairing due to the chosen coating. Characterised by flat annular contact surfaces which were pressed together with a pressure of 50 or $100 \mathrm{MPa}$, the samples were tested under alternating load with twisting angle amplitudes of $0.23^{\circ}$ and $0.5^{\circ}$ (or 46 and 100 microns slip, respectively). This technique enabled both optical damage (fretting, etc.) and strength-affecting mechanisms (damage of the substrate material) to be recorded. The results were evaluated using appropriate damage criterion, thereby identifying the accumulated dissipated friction energy as a fail-related physical quantity.

Keywords: fretting wear, friction energy, carbon-based coatings (incl. DLC), solid lubricant coatings, finite life.
\end{abstract}

\section{Introduction}

Fasteners, mechanical joints and couplings are typically subjected to dynamic service loading and/or vibration in a variety of machine parts. Due to the unequal elasticity and stiffness of the coupled bodies involved in such service loading, partial relative displacement within the contact area, i.e. slip, can occur. In addition, friction between the contact surfaces contributes to tribological damage in the form of fretting fatigue and wear. These phenomena are widely known to be causes of failure in many industrial applications, including aerospace, mechanical, civil, electrical and medical engineering [1, 2]. The friction 
behaviour of contact surfaces subjected to fretting loading is governed by several parameters. Besides the mechanical and material properties of the system components (contact bodies and coating), special attention must also be paid to local microscopic loading and associated stress characteristics. When considering tribological contact damage, other aspects arise in addition to component geometry, material stress and reliability. While the general contact environment, including lubricant (air, grease, oil, potable- or sea-water, etc.), is a crucial parameter influencing tribological durability, the coatings themselves can also have a positive impact on component and system life. Figure 1 represents a generalized structure of a typical coated contact pair. Both the design of the functional coatings and the resulting tribological performance depend very closely on the precise definition of system requirements [3]. A large amount of research has been carried out with the aim of describing the fretting behaviour of a variety of different coatings, with mixed results presented for the friction and wear behaviour of coatings and steel surfaces [4]. Frequently, the operating ranges of particular coatings and lubricants are restricted. However, such applications are often only investigated in terms of incomplete, i.e. Hertzian, contacts (point or line contact). Results obtained using only Hertzian contacts only moderately reflect typical contact geometries, such as those observed in flange and shaft-hub connections. For this reason, studies of fretting behaviour should include functional tests of the tribological characteristics involved under realistic operating conditions (complete contact). Leidich et al. [5] carried out a complete contact fretting test performed on newly-designed apparatus consisting of planar contact surfaces.

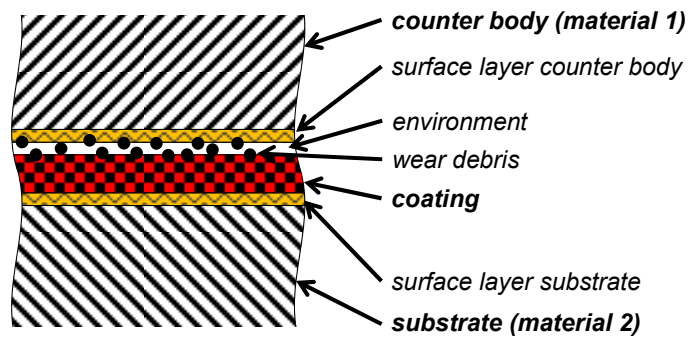

Figure 1: Schematic structure of a coated contact pair.

Besides the evaluation of experimental conditions, interpretation of the subsequently obtained data also plays an important role in tribological testing. Considering the manifold influences involved, a physical criterion is a useful tool with which to generalize these results. It is also essential that this criterion is transferable for use in typical user applications. Numerous methods have been developed specifically for such a purpose, including the energetic description of coating damage behaviour $[6,7]$, as well as those based on accumulated slip, the increase in the coefficient of friction [8] and relative changes in coating thickness [9]. However, these methods are not able to make a universally valid statement about the limits of the tribological wear resistance of a coating. 
Recently Maiwald et al. [10] verified two different contact pairs with the energy wear criterion as a quantitative parameter for assessing the wear resistance of the contact surface. These results will be extended in this study with additional coatings, to ensure a fundamental generalization of this criterion.

\section{Experimental specifications}

\subsection{Friction testing apparatus}

In order to obtain a realistic shear stress distribution and keep the specimen cost low, an annular geometry was chosen for the tested complete contact surface, as shown in fig. 2. Further details regarding the testing apparatus can be found in earlier publications $[11,12]$.

a)

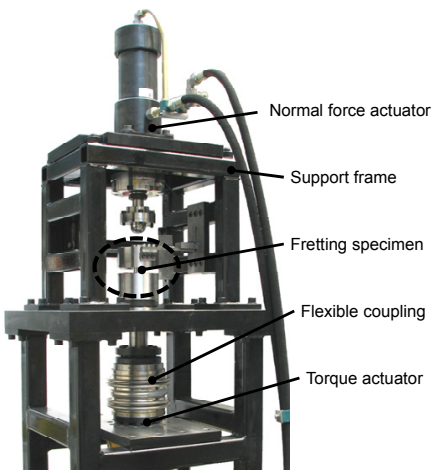

b)

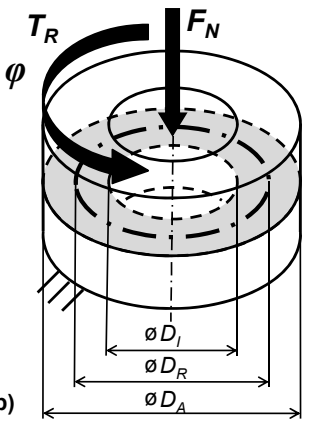

Figure 2: a) Friction testing apparatus; b) loading scheme and dimensions of the planar annular contact surface.

During the experimental procedure, two cylindrical specimens are pressed hydraulically with a normal force $F_{N}$, with torque $T_{R}$ applied to each in the form of friction (quasi-statically) and fretting loading (pulsating or alternating), respectively. A major advantage of this test method - in comparison to typical fretting test rigs involving Hertzian contact - is its ability to maintain a constant contact pressure distribution. Consequently, the required test surface pressure can be adjusted precisely, with the friction and wear results then better transferable to typical practical applications (e.g. shaft-hub-connections, bolted contact surfaces etc.). The above testing apparatus can be used in quasi-static friction testing to obtain a maximum value of sticking friction coefficient, as well as in the dynamic fretting testing of surface pairs. The dimensions of the contact surface can be varied up to a maximum outer diameter of $45 \mathrm{~mm}$. In the present study, annular fretting specimens of $D_{I}=15 \mathrm{~mm}$ and $D_{A}=30 \mathrm{~mm}$ were used. As mentioned previously, the fretting test was performed by applying dynamic torque to the lower specimen which leads to a dynamic angular displacement $\varphi$ between the specimens. In order to achieve a constant sliding amplitude 
throughout the test, an angular control system was applied to the hydraulic actuator.

\subsection{Measurement method}

The torque, normal load and relative angle between the specimens were measured constantly throughout the beginning of the test run. After the first 1000 cycles, measurements were carried out periodically every 1000 cycles in order to reduce the memory requirements of the experiment. During this transition the measurement was briefly interrupted. The fretting wear endurance of the applied coatings was tested at $22 \mathrm{~Hz}$ for 150,000 loading cycles. Temperature and humidity complied with standard laboratory environment values and were not controlled during the tests. All the fretting tests presented were carried out using normal loads of $F_{N}=26.5 \mathrm{kN}$, i.e. a nominal contact pressure of $50 \mathrm{MPa}$. The nominal angular slip amplitude was $\varphi=0.5^{\circ}$. In order to determine the effects of higher friction energy input, the slip amplitude and normal load were then adjusted to $\varphi=0.23^{\circ}$ and $F_{N}=53.0 \mathrm{kN}$. Using an effective friction diameter of the annular surface determined according to eqn. (1), $D_{R}=23.33 \mathrm{~mm}$, the applied slip amplitudes were $46 \mu \mathrm{m}$ and $100 \mu \mathrm{m}$, respectively.

$$
D_{R}=\frac{2 \cdot\left(D_{A}^{3}-D_{I}^{3}\right)}{3 \cdot\left(D_{A}^{2}-D_{I}^{2}\right)}
$$

\subsection{Specimen and coatings}

The specimens used in the friction tests are made of 34CrNiMo6 +QT (1.6582, $\mathrm{UTS}=1100+100 \mathrm{MPa}$ ) and $16 \mathrm{MnCr} 5 \mathrm{E}(1.7131$, hardness $59 \mathrm{HRC})$. In each case the surface of the specimen, consisting of 34CrNiMo6 +QT (1.6582), was treated with the anti-fretting coating. In order to represent hard thin films (thickness $<10$ microns), a DLC coating was considered. A CrC coating was chosen to analyse a hard thick film (thickness approx. 100 microns). To represent soft layers, a $\mathrm{MnPh}$ coating was selected for thin films (10 micron layer) and a Zn-coating for thick films (thickness approx. 60 microns). An uncoated contact pair was also tested as a reference.

\subsection{Evaluation method}

\subsubsection{Maximum cyclic friction coefficient}

The tests were primarily evaluated in terms of the resultant friction behaviour. The actual coefficient of friction (COF) can be calculated using eqn. (2) depending on the measured normal force resp. torque and surface geometry (see also fig. $2 \mathrm{~b}$ ).

$$
\mu=\frac{2 \cdot T_{R}}{D_{R} \cdot F_{N}}
$$

Assuming a homogeneous contact pressure distribution, the friction diameter was $D_{R}=23.33 \mathrm{~mm}$, with the final result then representing the maximum 
coefficient of friction per fretting cycle. The maximum achievable value can either be the static friction peak amplitude (fig. 3, left), or the final value of a load cycle (sliding friction; fig. 3, right). Moreover, the maximum COF can be in either the positive or negative torque region of the load cycle. As a result, the absolute value is evaluated on every occasion (eqn. (3)).

$$
\mu_{Z, \max }=\left|\frac{2 \cdot T_{R, Z, \max }}{D_{R} \cdot F_{N}}\right|
$$

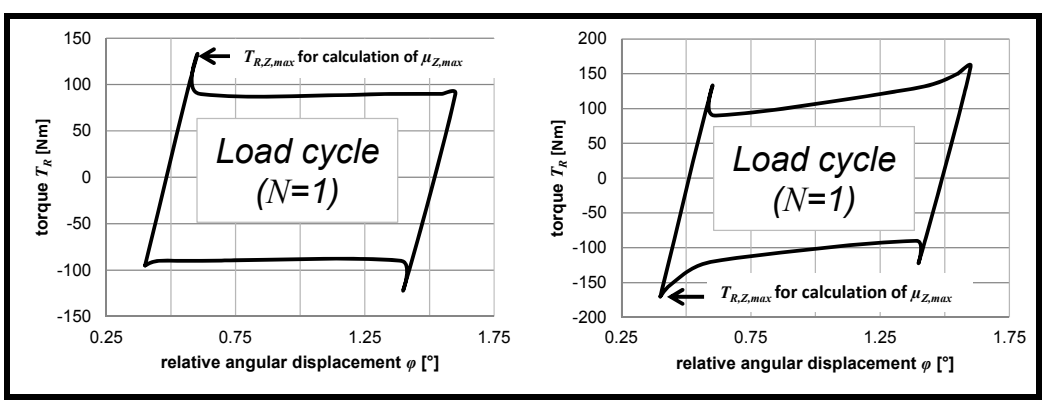

Figure 3: $\quad$ Evaluation of maximum torque $T_{R, Z, \max }$ depending on torque regime (left: static friction peak amplitude; right: final value of a load cycle).

\subsubsection{Accumulated specific dissipated friction energy}

In the characterisation and quantitative evaluation of surface damage in fretted surfaces, the dissipated friction energy $W_{\text {fric }}$ is an important physical quantity. $W_{\text {fric }}$ is generally defined as the integral of frictional force $F_{R}$ on the applied relative angular slip $s_{R}$, as in eqn. (4).

$$
W_{\text {fric }}=\oint F_{R} \cdot d s_{R}
$$

However, for the experimental determination of friction energy, eqn. (4) must be modified according to the contact surfaces tested, as in eqn. (5). Figure 3 shows two examples of recorded hysteresis (torque $T_{R}$ on the relative angular displacement $\varphi$ ). The inner area of the hysteresis loop represents the dissipated friction energy of the load cycle analysed.

$$
W_{\text {fric }}=\oint T_{R} \cdot d \varphi=\oint \frac{D_{R}}{2} \cdot F_{R} \cdot \frac{1}{D_{R}} d s_{R}
$$

Consequently, the accumulated friction energy is thus calculated depending on the number of load cycles. To represent a specific (i.e. contact surface

area-independent) value, the friction energy is divided by the surface area $A$, as in eqn. (6). 


$$
W_{\text {fric }, a c c}=\frac{1}{A} \sum_{n=1}^{N} W_{\text {fric }}(n)
$$

\section{Analysis - tribological wear criterion}

The aim of the present work was to define a physically-based criterion able to express a universally valid wear-description of the surface resp. coating under various tribological conditions, such as slip amplitude, contact pressure etc. Examining the results of the completed friction experiments, the criterion based on the accumulated specific friction energy was successfully verified, as will be shown later. Figure 4 shows the variation in friction behaviour (max. COF) with increasing accumulated specific friction energy $W_{\text {fric,acc }}$ for the DLC-coated contact surface.

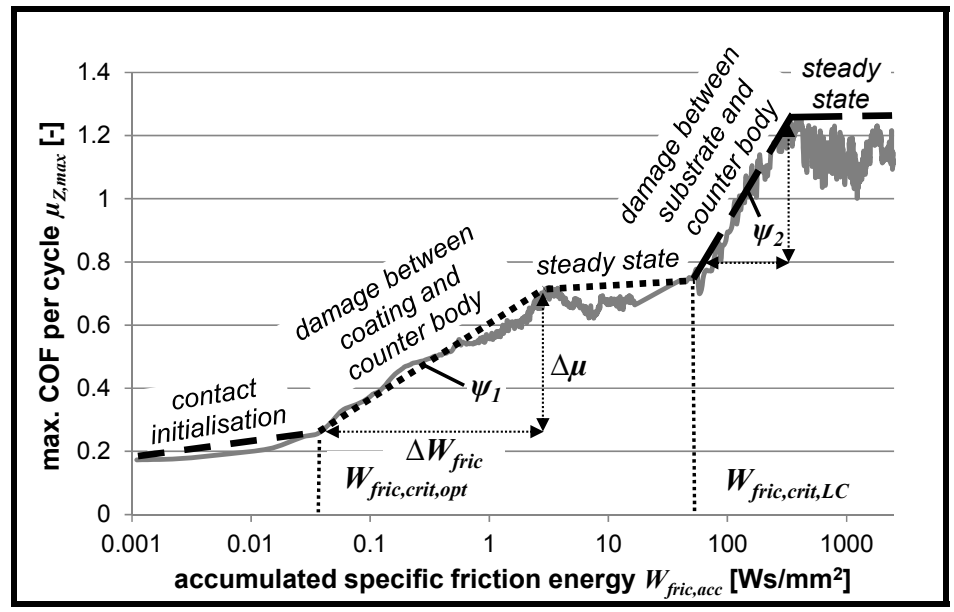

Figure 4: Selected example describing the different phases of a coated contact pair and the critical characteristic values (wear criterion).

The regime can be divided into distinct phases highlighted by the different trend lines. At the beginning of the experiment - contact initialisation - no optical damage occurs on the coating. In phase two, the linear-logarithmic slope $\psi_{1}=\Delta \mu / \Delta W_{\text {fric }}$ of the coefficient of friction is clearly visible; during this phase the coating / counter surface is continually damaged. The subsequent drop in the trend line in phase three represents the complete damage of the coating, i.e. steady-state linear wear of the contact pair. Following this, damage also occurs between the substrate and counter body (linear-logarithmic slope $\psi_{2}$ ), which leads to a final steady-state phase. Evaluation of the experimental results reveals that damage to the contact surface is directly related to the friction energy introduced. The absolute critical energy input $W_{\text {fric,crit }}$ is therefore the limiting criterion for the respective material combination, and thus also depends on the contact partners involved: 
- counter body vs. coating (relevant for optical damage such as wear debris, i.e. fretting corrosion) $W_{\text {fric, crit,opt }}$

- counter body vs. substrate (relevant for protection against the consequences of fretting) $W_{\text {fric, crit }, L C}$

Typical forms of wear which are spread homogeneously over the entire contact area are thus determined to be critical for optical damage $W_{\text {fric,critopt }}$ to friction surfaces. In contrast, the coating is critical for protection against the consequences of fretting, with even a small partial contact between the substrate and the surface of the counter body despite an otherwise intact coating sufficient to represent a failure criterion $\left(W_{\text {fric, crit,LC }}\right)$.

\section{Results}

\subsection{Uncoated contact}

In this section the test results are analysed in terms of the tribological wear criterion, with the specimen surface condition after testing illustrated using optical analysis methods. Figure 5 shows the friction regimes of the uncoated contact pairs for three different values of the experimental parameters ( $p$ and $\varphi)$. There is a clear uniform limit with regard to damage of the substrate, at $W_{\text {fric, crit }, L C}=0.24 \mathrm{Ws} / \mathrm{mm}^{2}$. The averaged linear-logarithmic slope $\psi_{2}$ is 0.56 .

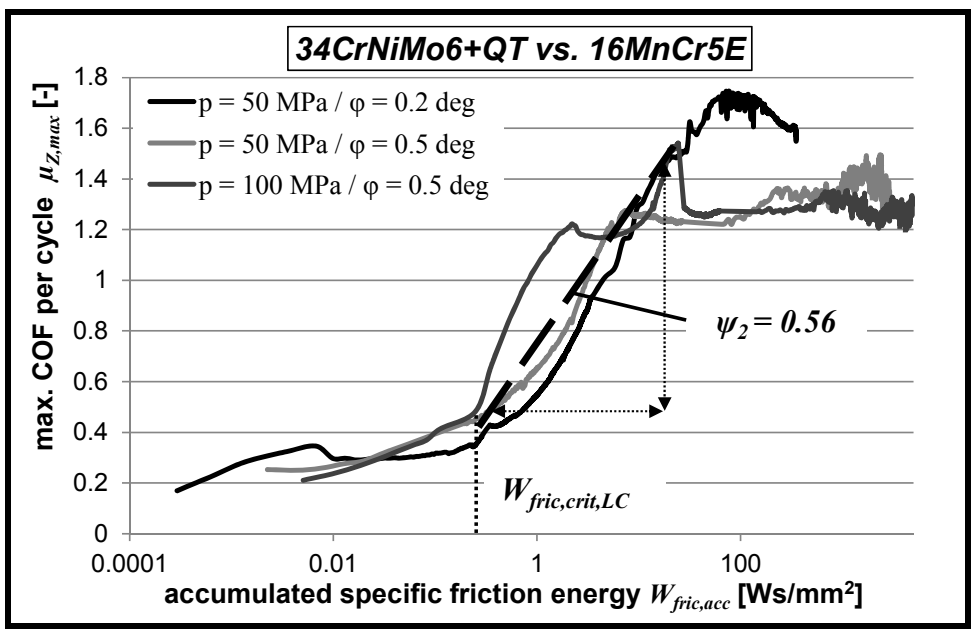

Figure 5: Variation in max. COF per cycle with increasing accumulated specific friction energy for the uncoated contact pair, for different values of contact pressure $p$ and nominal angular slip amplitude $\varphi$.

\subsection{Hard coatings}

Figure 6 shows the friction regimes of the DLC-coated contact pair for three different values of the experimental parameters. Two uniform limits are 
apparent, the first with regard to the wear of the contact surfaces; $W_{\text {fric, crit,opt }}=0.05 \mathrm{Ws} / \mathrm{mm}^{2}$, and a second associated with the damage of the substrate; $W_{\text {fric, crit } L C}=28 \mathrm{Ws} / \mathrm{mm}^{2}$. Furthermore, a significant difference can be observed between slope $\psi_{1}=0.22$ (wear between counter body vs. coating) and $\psi_{2}=0.70$ (wear between counter body vs. substrate). Slope $\psi_{2}$ and the friction level in the steady state $\mu_{Z, \max } \approx 1.2$ are comparable to that of fig. 5 (uncoated contact pair).

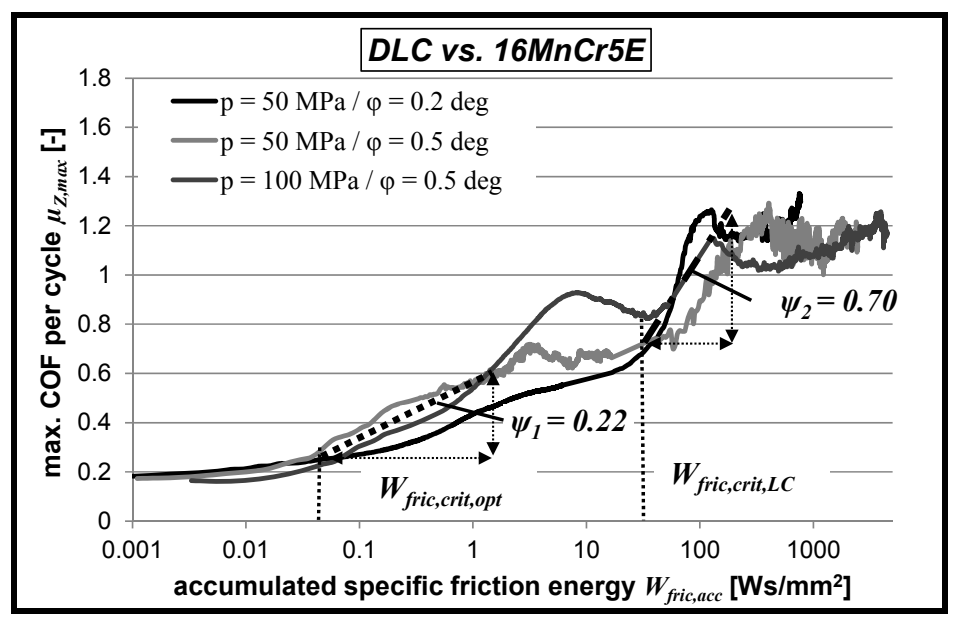

Figure 6: Variation in max. COF per cycle with increasing accumulated specific friction energy for the DLC-coated contact pair, for three different values of contact pressure $p$ and nominal angular slip amplitude $\varphi$.

Figure 7 shows the friction regimes of the $\mathrm{CrC}$-coated contact pair for three different values of the experimental parameters. The two uniform limits are again apparent $\left(W_{\text {fric, crit,opt }}=0.024 \mathrm{Ws} / \mathrm{mm}^{2}, \quad W_{\text {fric, crit } L C}=22 \mathrm{Ws} / \mathrm{mm}^{2}\right)$. In this case slopes $\psi_{1}=0.43$ and $\psi_{2}=0.39$ are nearly identical. Slope $\psi_{2}$ and the friction level in the steady state $\mu_{Z, \max } \approx 1.4$ is comparable to that of fig. 5 (uncoated contact pair). Figure 8 shows the friction regimes of the $\mathrm{MnPh}$ coated contact pairs for three different values of the experimental parameters. The two uniform limits are again apparent $\left(W_{\text {fric, crit,opt }}=0.013 \mathrm{Ws} / \mathrm{mm}^{2}, W_{\text {fric, crit }, L C}=12 \mathrm{Ws} / \mathrm{mm}^{2}\right)$. So the passable friction energy $W_{\text {fric,crit } L C}$ is significantly lower in contrast to the hard coatings. For the $\mathrm{MnPh}$ coating a significant difference between slope $\psi_{1}=0.21$ and $\psi_{2}=0.54$ can be observed. Slope $\psi_{2}$ and the friction level in the steady state $\mu_{Z, \max } \approx 1.2$ are again comparable to that of fig. 5 (uncoated contact pair). 


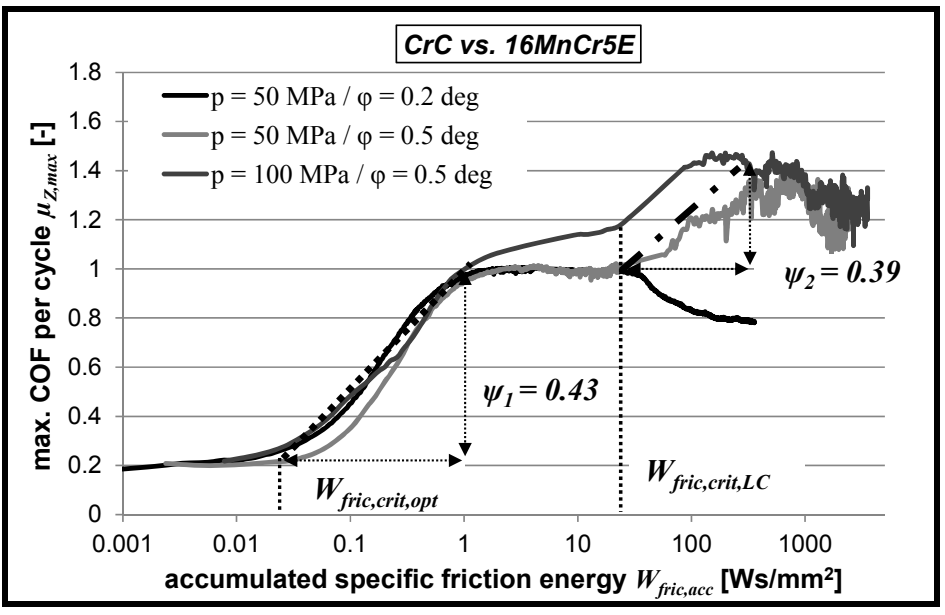

Figure 7: Variation in max. COF per cycle with increasing accumulated specific friction energy for the $\mathrm{CrC}$-coated contact pair, for three different values of contact pressure $p$ and nominal angular slip amplitude $\varphi$.

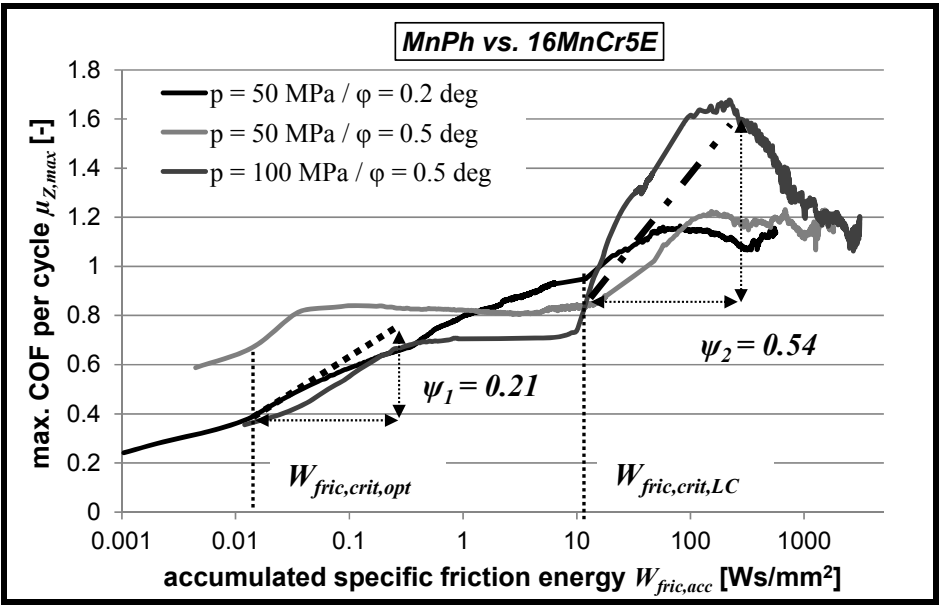

Figure 8: Variation in max. COF per cycle with increasing accumulated specific friction energy for the solid film lubricant-coated contact pair, for two different values of contact pressure $p$.

\subsection{Soft coatings}

Figure 8 shows the friction regimes of the $\mathrm{MnPh}$-coated contact pairs for three different values of the experimental parameters. The two uniform limits are again 
apparent $\left(W_{\text {fric, crit opt }}=0.013 \mathrm{Ws} / \mathrm{mm}^{2}, W_{\text {fric, crit } L C}=12 \mathrm{Ws} / \mathrm{mm}^{2}\right)$. So the passable friction energy $W_{\text {fric,crit } L C}$ is significantly lower in contrast to the hard coatings. As a consequence, the $\mathrm{MnPh}$ coating has a minor mechanical resilience under fretting conditions in comparison to the hard coatings tested. For the $\mathrm{MnPh}$ coating a significant difference between slope $\psi_{1}=0.21$ and $\psi_{2}=0.54$ can be observed. Slope $\psi_{2}$ and the friction level in the steady state $\mu_{Z, \max } \approx 1.2$ are again comparable to that of fig. 5 (uncoated contact pair).

Figure 9 shows the friction regimes of the $\mathrm{Zn}$-coated contact pairs for three different values of the experimental parameters. The two uniform limits are again apparent $\left(W_{\text {fric, crit,opt }}=0.09 \mathrm{Ws} / \mathrm{mm}^{2}, W_{\text {fric, crit } L C}=46 \mathrm{Ws} / \mathrm{mm}^{2}\right)$. That means that the $\mathrm{Zn}$ coating can dissipate the greatest amount of energy in comparison to the other coatings tested. For the $\mathrm{Zn}$ coating a significant difference between slope $\psi_{1}=0.32$ and $\psi_{2}=0.62$ can be observed. Slope $\psi_{2}$ and the friction level in the steady state $\mu_{Z, \max } \approx 1.3$ are again comparable to that of fig. 5 (uncoated contact pair).

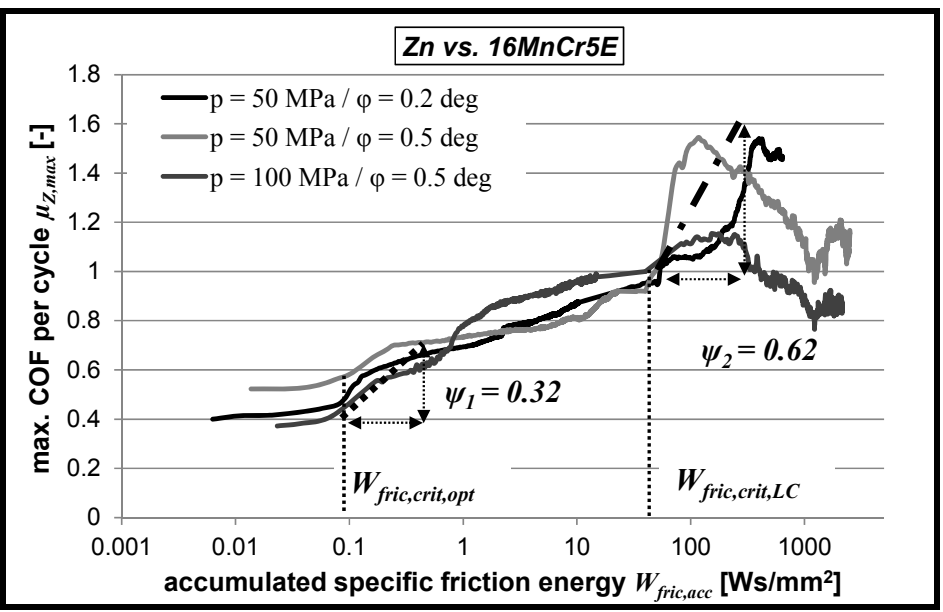

Figure 9: Variation in max. COF per cycle with increasing accumulated specific friction energy for the solid film lubricant-coated contact pair, for two different values of contact pressure $p$.

\section{Application of results}

Future work will be directed towards the practical application of these criteria and associated limits. In order to carry out such work the application-specific slip range and contact pressure independent of real friction behaviour must be calculated. For this purpose, extensive 3D finite element simulations are currently the only viable technique available.

When classifying the calculated values for use in typical applications, such as shaft hub connections, it should be noted that shrink-fitted shaft hub connections dissipate a relatively minor amount of frictional work. This is due to the much 
smaller slip amplitudes resulting from bending or torque (5 to 20 microns) in comparison to the experimental conditions applied in the present study. With possible friction coefficients of $\mu=0.7$ to 1 in the edge array of a hub in many cases, a slip-free joint can be assumed. It may therefore be expected that the measured critical friction energy with regard to the damage of a substrate of $W_{\text {fric,crit } L C .} \approx 1-10 \mathrm{Ws} / \mathrm{mm}^{2}$ is similar to the damage inception point of the substrate of typical press fits at $N=10^{7}-10^{8} \mathrm{LC}$ [13]. However, further study is required in order to accurately evaluate this relationship.

\section{Conclusion}

Test bench experiments for the determination of friction coefficients using a standardized torsion test method were performed. The tested samples were characterised by flat annular contact surfaces which were pressed together and alternating loaded with defined twisting angle amplitudes. Two criteria based on the accumulated friction energy were devised to describe the damage stages observed during the testing of selected coated surfaces. The absolute critical energy input $W_{\text {fric,crit }}$ is therefore the limiting criterion for the respective material combination, which depends on the damage contained contact partners:

- counter body vs. coating (relevant for optical damage such as wear debris i.e. fretting corrosion) $W_{\text {fric,crit,opt }}$

- counter body vs. substrate (relevant for protection against the consequences of fretting) $W_{\text {fric, crit }, L C}$

In addition, the linear-logarithmic slope of the coefficient of friction $\psi$ was also evaluated, with a significant difference detected between slope $\psi_{1}$ (wear between counter body vs. coating) and $\psi_{2}$ (wear between counter body vs. substrate). Slope $\psi_{2}$ was seen to be comparable for initially uncoated and coated contact pairs. In addition, the friction coefficient in the second steady state $\mu_{Z, \max } \approx 1.3$ tallies. Finally, the transferability of the results to practical applications was discussed.

\section{Acknowledgements}

The authors would like to thank PHM Industrieanlagen $\mathrm{GmbH}$ and Pro-beam Technologies $\mathrm{GmbH}$ for the preparation of the modified DLC-coating.

\section{References}

[1] Hills, D.A. and Mugadu, A., An overview of progress in the study of fretting fatigue. Journal of Strain Analysis, 37(6), pp. 591-601, 2002.

[2] Nowell, D., Dini, D. and Hills, D.A., Recent developments in the understanding of fretting fatigue. Engineering Fracture Mechanics, 73(2), pp. 207-222, 2007.

[3] Lampke, Th., Gestaltung Technischer Oberflächen mit Funktionalen Aufgaben. Habilitation, Chemnitz University of Technology, 2008. 
[4] Stachowiak, G.W., Batchelor, A.W. and Stachowiak, G.B., Experimental Methods in Tribology, Tribology Series Vol. 44. Elsevier: Amsterdam, 2004.

[5] Leidich, E., Schuller, S., Vidner, J., Meyer, M. and Gorodnev, A., Testing of tribological functionality of nano-scaled fillers in coatings preventing fretting fatigue. Proceedings of the 2nd Werkstofftechnisches Kolloquium. Chemnitz, 2009.

[6] Fouvry, S., Liskiewicz, T. and Paulin, C., A global-local wear approach to quantify the contact endurance under reciprocating-fretting sliding conditions. Wear 263, pp. 518-531, 2007.

[7] Lu, Z., Wang, L., Zhang, G. and Xue, Q., The dependence of energy dissipation on the elastic energy density of friction pairs in hard coating films. Tribology Letters, 41(2), 2011, DOI: 10.1007/s11249-010-9725-y.

[8] Eriten, M., Polycarpou, A.A. and Bergman, L.A., Surface roughness effects on energy dissipation in fretting contact of nominally flat surfaces. Journal of Applied Mechanics, 78, pp. 021011/1-8, 2011.

[9] Kyungmok, K., Analysis of friction coefficient evolutions on coated systems under sliding conditions. Wear, 269, pp. 655-663, 2010.

[10] Korsunsky, A.M., Torosyan, A.R. and Kim, K., Development and characterization of low friction coatings for protection against fretting wear in aerospace components. Thin Solid Films, 516, pp. 5690-5699, 2008.

[11] Maiwald, A., Leidich, E., Vidner, J. and Meyer, M., Energiebasiertes Schädigungskriterium zur quantitativen Beurteilung des Verschleißes infolge Fretting im ebenen Kontakt. Proceedings of the 52nd Tribologietagung der Gesellschaft für Tribologie e.V. Göttingen, 2011 (in German).

[12] Leidich, E. and Schuller, S., Haftreibung. Abschlussbericht FVV-Heft 906. Forschungsvereinigung Verbrennungskraftmaschinen: Frankfurt/M., 2010 (in German).

[13] Leidich, E. and Schuller, S., Referenzierte Haftreibungszahlen an Stirnflächenkontakten. VDI-Berichte, 2114, pp. 63-80, 2010 (in German).

[14] Leidich, E. and Maiwald, A., Benchmark Fretting. Abschlussbericht FVAHeft 994, Forschungsvereinigung Antriebstechnik: Frankfurt/M., 2011 (in German). 